\title{
Article \\ Genomic Insights into a Colistin-Resistant Uropathogenic Escherichia coli Strain of O23:H4-ST641 Lineage Harboring mcr-1.1 on a Conjugative IncHI2 Plasmid from Egypt
}

\author{
Azza S. Zakaria *(D), Eva A. Edward (D) and Nelly M. Mohamed (D) \\ Microbiology and Immunology Department, Faculty of Pharmacy, Alexandria University, \\ Alexandria 21500, Egypt; eve.farid@alexu.edu.eg (E.A.E.); nelly.mohamed@alexu.edu.eg (N.M.M.) \\ * Correspondence: azza.hanafy@alexu.edu.eg
}

check for updates

Citation: Zakaria, A.S.; Edward, E.A.; Mohamed, N.M. Genomic Insights into a Colistin-Resistant Uropathogenic Escherichia coli Strain of O23:H4-ST641 Lineage Harboring mor-1.1 on a Conjugative IncHI2 Plasmid from Egypt. Microorganisms 2021, 9, 799. https://doi.org/10.3390/ microorganisms 9040799

Academic Editor: Carlo Pazzani

Received: 26 March 2021

Accepted: 7 April 2021

Published: 10 April 2021

Publisher's Note: MDPI stays neutral with regard to jurisdictional claims in published maps and institutional affiliations.

Copyright: (C) 2021 by the authors. Licensee MDPI, Basel, Switzerland. This article is an open access article distributed under the terms and conditions of the Creative Commons Attribution (CC BY) license (https:// creativecommons.org/licenses/by/ $4.0 /)$.

\begin{abstract}
The reintroduction of colistin, a last-resort antibiotic for multidrug-resistant pathogens, resulted in the global spread of plasmid-mediated mobile colistin resistance $(\mathrm{mcr})$ genes. Our study investigated the occurrence of colistin resistance among Escherichia coli isolated from patients with urinary tract infections admitted to a teaching hospital in Egypt. Out of 67 isolates, three isolates were colistin-resistant, having a minimum inhibitory concentration of $4 \mu \mathrm{g} / \mathrm{mL}$ and possessing the $m c r-1$ gene. A double mechanism of colistin resistance was detected; production of $m c r-1$ along with amino acid substitution in PmrB (E123D and Y358N) and PmrA (G144S). Broth mating experiments inferred that $m c r-1$ was positioned on conjugative plasmids. Whole-genome sequencing of EC13049 indicated that the isolate belonged to O23:H4-ST641 lineage and to phylogroup D. The mcr-1-bearing plasmid corresponded to IncHI2 type with a notable similarity to other E. coli plasmids previously recovered from Egypt. The unbanned use of colistin in the Egyptian agriculture sector might have created a potential reservoir for the $m c r-1$ gene in food-producing animals that spread to humans. More proactive regulations must be implemented to prevent further dissemination of this resistance. This is the first characterization of mcr-1-carrying IncHI2:ST4 plasmid recovered from E. coli of a clinical source in Egypt.
\end{abstract}

Keywords: colistin resistance; $m c r-1$; multidrug resistant uropathogenic E. coli; IncHI2 plasmid; whole genome sequencing; Egypt

\section{Introduction}

Urinary tract infections (UTIs) have been categorized amongst the most frequently encountered pathological conditions affecting 150 million people around the globe every year [1]. Although other bacteria belonging to the family Enterobacteriaceae can cause UTIs, uropathogenic Escherichia coli (UPEC) arises as the predominant etiologic agent responsible for more than $80 \%$ of UTI cases universally [2]. The emergence of a multidrug-resistant (MDR) phenotype among UPEC over recent decades worldwide is alarming and has been strongly correlated with the inappropriate empiric antimicrobial therapy [3]. Due to this escalating problem of the widespread of MDR UPEC pathogens, coupled with the exhausted antibiotic invention pipeline, colistin has been reintroduced into clinical practice after being classified by the WHO as one of the antibiotics of critical importance in human clinical settings [4]. Colistin is a polycationic peptide capable of binding to the anionic lipopolysaccharides located in the outer membrane of the Gram-negative cell wall, thus resulting in cell lysis [5]. Intrinsic colistin resistance has been linked to chromosomal mutations in the genes encoding the PmrA/PmrB and PhoP/PhoQ twocomponent systems or the negative regulator $\mathrm{MgrB}$ resulting in alterations of the lipid A molecule, the principal target of colistin [6]. In 2015, a plasmid-mediated colistin resistance gene, $m c r-1$, encoding phosphoethanolamine transferase, was identified on a conjugative IncI2 plasmid in China [7]. To date, $m c r-1$ gene has been found carried on IncI2, IncX4, IncFI, 
IncFII, IncFIB, IncHII, IncHI2, IncP, and IncY plasmid types [8], accelerating its transmission between different bacterial species. In addition, this gene could easily disseminate from livestock, where colistin is used as a treatment or as a growth promoter in food-animal production, to humans through horizontal gene transfer [9]. The irresponsible use of colistin in veterinary practice, especially in the absence of strict legislations has contributed to the global spread of the $m c r-1$ gene in $10 \%$ of animal isolates and in $0.1-2 \%$ of human isolates [5]. Despite ample information on the distribution of $m c r-1$ gene worldwide, very few studies have investigated the prevalence of this gene in E. coli of human origin in Egypt where two mor-1-postive E. coli strains from clinical settings have been reported so far $[10,11]$. In the present study, we aimed to shed light on the occurrence of colistin resistance among E. coli isolated from patients with UTIs in Alexandria, Egypt and to investigate the underlying mechanisms of this resistance. Additionally, we are describing the genomic features of an MDR UPEC strain belonging to ST641 clone and O23:H4 serotype, harboring the mcr1.1 gene on an IncHI2 plasmid. This is the first complete sequence of $m c r-1$-carrying IncHI2 plasmid recovered from an E. coli strain with a clinical source in Egypt.

\section{Materials and Methods}

\subsection{Bacterial Strains Collection and Identification}

A total of 67 E. coli clinical isolates were collected through the routine laboratory facility of Alexandria Main University Hospital (AMUH) from the urine cultures of patients admitted to the hospital with UTIs over the period of June to December 2019. AMUH is a 1500-bed main referral hospital in the northern sector of Egypt with approximately 100,000 total hospital admissions per year. Samples were streaked onto MacConkey (Oxoid) and eosin methylene blue (Oxoid, Hampshire, UK) agar plates. Following incubation at $37^{\circ} \mathrm{C}$ for $24 \mathrm{~h}$, the isolated colonies were identified by Gram staining then subjected to standard biochemical tests including triple-sugar iron, citrate utilization and urease tests. The colistin-resistant isolates were further identified by the Vitek ${ }^{\circledR} 2$ Advanced Expert System $^{\mathrm{TM}}$ (BioMérieux, La-Balme-les-Grottes, France).

\subsection{Antimicrobial Susceptibility Testing}

The susceptibility of the 67 isolates to amoxicillin-clavulanate, cefepime, cefotaxime, ceftazidime, ciprofloxacin, levofloxacin, gentamicin, imipenem, meropenem, doxycycline, sulfamethoxazole-trimethoprim and colistin was determined by the disk diffusion method. The results were interpreted according to Clinical Laboratory Standards Institute (CLSI, 2020), except for colistin, where the disk manufacturer's guidelines (Oxoid, Hampshire, UK) were used to interpret the results [12]. The phenotype of the E. coli isolates was defined as MDR based on the International Expert proposal for Interim Standards Guidelines [13]. Minimum inhibitory concentration (MIC) of colistin (colistin sulfate, Sigma Chemical, St. Louis, USA) against the collected $67 \mathrm{E}$. coli isolates was detected by the broth microdilution in triplicates using cation-adjusted Muller-Hinton broth (Difco-BBL, Detroit, MI, USA) in accordance with the protocols recommended in M100-S30 of the 2020 CLSI which considered isolates with a colistin MIC value of $\geq 4 \mu \mathrm{g} / \mathrm{mL}$ to be resistant [12]. The E. coli ATCC 25922 was included as a quality control strain.

\subsection{Detection of Antibiotic Resistance Genes by Polymerase Chain Reaction (PCR)}

E. coli isolates with a colistin MIC value of $>2 \mu \mathrm{g} / \mathrm{mL}$ were screened using PCR for the presence of mobile colistin resistance genes, $m c r-1$ and $m c r-2$ [14], and the following chromosomally encoded genes related to colistin resistance: $p m r A, p m r B, P h o P, P h o Q, m g r B$ and $p m r D$ [15]. Other plasmid-borne genes conferring resistance to extended-spectrum $\beta$-lactams such as bla $a_{S H V}$ and $b l a_{T E M}$ [16], carbapenems, bla $a_{N D M}$ [17], and fluoroquinolones, qnrB [16], were analyzed as well. The sequences of the used primers obtained from Willowfort, UK, are summarized in Table 1. Genomic DNA from colistin-susceptible E. coli ATCC 25922 was used as the negative control. The sizes of the PCR products were determined by comparison with a molecular-sized standard (GeneRuler ${ }^{\mathrm{TM}} 1 \mathrm{~kb}$ and 
100 bp DNA ladder, Thermo Fisher Scientific, Hampshire, UK). Amplified DNA fragments corresponding to $\operatorname{pmr} A, \operatorname{pmr} B, \mathrm{PhoP}, \mathrm{PhoQ}, m g r B$ and $\operatorname{pmrD}$ were purified using Zymo Research $^{\mathrm{TM}}$ DNA Purification Kit and sequenced in both directions using a system from LGC Co. Ltd. (Berlin, Germany). Mutations were determined by alignment with reference genome E. coli K-12 MG1655 (\# NC_000913.3648.1) using NCBI BlastX tool.

Table 1. List of primer pairs used for PCR amplification of the selected genes in this study.

\begin{tabular}{|c|c|c|c|}
\hline Target Gene & Nucleotide Sequence $\left(5^{\prime} \rightarrow 3^{\prime}\right)$ & Amplicon Size (bp) & Reference \\
\hline$m c r-1$ & $\begin{array}{l}\text { F: 5'-CGGTCAGTCCGTTTGTTC-3' } \\
\text { R: 5'-CTTGGTCGGTCTGTAGGG-3 }\end{array}$ & 309 & [14] \\
\hline$m c r-2$ & $\begin{array}{l}\text { F: 5'-TGTTGCTTGTGCCGATTGGA-3' } \\
\text { R: 5'-AGATGGTATTGTTGGTTGCTG-3' }\end{array}$ & 567 & [14] \\
\hline$m g r B$ & $\begin{array}{l}\text { F: } 5^{\prime} \text {-TCGTAGCGGCAATATGCGC-3' } \\
\text { R: } 5^{\prime}-\text {-GACGCAATGTTCATCACGCC-3' }\end{array}$ & 385 & [15] \\
\hline PhoP & $\begin{array}{l}\text { F: } 5^{\prime} \text {-ATGCGCGTACTGGTTGTTG-3' } \\
\text { R: } 5^{\prime} \text {-AGTACTACCGCTGCCGTTGCC-3' }\end{array}$ & 747 & [15] \\
\hline PhoQ & $\begin{array}{l}\text { F: 5'-CAGGGCTATCTGTTCGAATTGCG-3' } \\
\text { R: 5'-ACGGATGCTTAACGTAATGCGTG-3' }\end{array}$ & 1523 & [15] \\
\hline pmrA & $\begin{array}{l}\text { F: 5'-CTGATTGTTGAAGACGATACGC-3' } \\
\text { R: 5'-AGTTTTCCTCATTCGCGACCA-3' }\end{array}$ & 657 & [15] \\
\hline$p m r B$ & $\begin{array}{c}\text { F: } 5^{\prime} \text {-GAATCTGATGCATTTGCGC-3' } \\
\text { R: } 5^{\prime} \text {-TTATATCTGGTTGCCACGTACTG-3' }\end{array}$ & 1098 & [15] \\
\hline pmrD & $\begin{array}{l}\text { F: 5'-CGGTGCGCTGGCTAACTCTGCC-3' } \\
\text { R: 5'-AGTAGCCTGTATTATGAGCGGG-3'3 }\end{array}$ & 527 & [15] \\
\hline$b l a_{S H V}$ & $\begin{array}{l}\text { F: 5'-ATT TGT CGC TTCTTT ACT CGC -3' } \\
\text { R: 5'-TTT ATG GCG TTACCT TTG ACC-3' }\end{array}$ & 1018 & [16] \\
\hline$b l a_{T E M}$ & $\begin{array}{l}\text { F: 5'-CATTTCCGTGTCGCCCTTATTC-3' } \\
\text { R: 5'-CGTTCATCCATAGTTGCCTGAC-3' }\end{array}$ & 800 & [16] \\
\hline$q n r B$ & $\begin{array}{l}\text { F: 5'-CCT GAGCGGCACTGAATTTAT-3' } \\
\text { R: } 5^{\prime} \text {-GTT TGCTGCTCGCCAGTCGA -3' }\end{array}$ & 120 & [16] \\
\hline$b l a_{N D M}$ & $\begin{array}{l}\text { F: } 5^{\prime} \text {-GGTTTGGCGATCTGGTTTTC- } 3^{\prime} \\
\text { R: } 5^{\prime}-C G G A A T G G C T C A T C A C G A T C-3^{\prime}\end{array}$ & 621 & [17] \\
\hline
\end{tabular}

\subsection{Conjugation Experiment}

Transferability of plasmid by conjugation was determined by mating Luria-Bertani (Oxoid, Hampshire, UK) broth cultures of colistin-resistant donor strains with rifampicinresistant E. coli $\mathrm{K}-12$ recipient at $37^{\circ} \mathrm{C}$ and incubating overnight. Transconjugants were selected on MacConkey agar (Oxoid, Hampshire, UK)) supplemented with rifampicin $(100 \mu \mathrm{g} / \mathrm{mL})$ and colistin $(4 \mu \mathrm{g} / \mathrm{mL})$ [18] then tested for susceptibility to previously mentioned antimicrobials. The MIC of colistin for recipient and transconjugants was determined using the broth microdilution method as described earlier. The presence of $m c r-1$ and the co-transfer of other resistance genes $\left(b l a_{S H V}, b l a_{T E M}, b l a_{N D M}\right.$ and $\left.q n r B\right)$ were confirmed by PCR amplification using extracted plasmids (QIAGEN Plasmid Mega Kit, Netherlands) as DNA templates and the previously mentioned primers.

\subsection{Whole Genome Sequencing (WGS) and Bioinformatics Tools}

Genomic DNA was extracted from overnight culture of E. coli strain EC13049 using an Invitrogen Easy-DNA ${ }^{\mathrm{TM}}$ kit (Invitrogen, San Diego, CA, USA), and DNA concentration was determined using the Qubit ${ }^{\mathrm{TM}}$ dsDNA BR assay kit (Invitrogen, San Diego, CA, USA). The genomic DNA was prepared for Illumina pair-end sequencing using the Illumina Nextera XT DNA Library Prep Guide Document \# 15031942 v05 May 2019 following the protocol (Nextera XT DNA Library Prep Kit Reference Guide (15031942) (illumina.com)) (accessed on 18 August 2020)). The library was sequenced on an Illumina MiSeq using MiSeq Reagent Kit v2 and 500 cycles with a Standard Flow Cell. The raw data were demultiplexed using Illumina's bcl2fastq tool (Illumina, San Diego, CA, USA), checked for quality using the FastQC tool (BaseSpace, Illumina, San Diego, CA, USA) and then quality-trimmed (Q25) and adapter-trimmed (multiplexing and sequencing adapters) using the FastQ Toolkit (BaseSpace, Illumina, CA, USA). The raw reads were assembled using the Assembler pipeline (version 1.2) available from the Center for Genomic Epidemiology (CGE) (https: / / cge.cbs.dtu.dk/services / Assembler/) (accessed on 21 October 2020), which is based on 
the Velvet algorithms for de novo short reads assembly. The assembled sequences were analyzed to confirm the species and serotype of E. coli strain using the CGE pipelines; K-merResistance (version 2.2) and SeroTypeFinder (version 2.0). Following confirmation, the MLST sequence type (ST), plasmid replicons, and acquired antimicrobial resistance (AMR) genes were identified using the pipelines: MLST (version 2.0), PlasmidFinder (version 2.0), and ResFinder (version 4.0) with 95\% identity and 60\% minimum alignment length as thresholds, available as well from CGE. The generated contigs were annotated using NCBI Prokaryotic Genome Annotation Pipeline (PGAP) (NCBI Prokaryotic Genome Annotation Process (nih.gov) (accessed on 29 January 2021). Phylogrouping was based on analysis using Clermont typing [19]. To determine the mcr-1-harbouring plasmid sequence, assembled contigs from E. coli EC13049 were mapped against Escherichia coli (taxid:562) using BlastN (https:/ / blast.ncbi.nlm.nih.gov/Blast.cgi): (accessed on 4 December 2020). The presence of the $m c r-1.1$ gene was identified in node 365 in the assembled contigs and the complete sequence of the generated plasmid pEGY49_MCR1.1 in E. coli strain EC13049 was assembled by scaffolding several nodes while any overlap regions were manually inspected. The assembled contigs were compared to the sequences of plasmids pHNSHP452 (GenBank accession number KU341381), pEGY1-MCR-1 (GenBank accession number CP023143) and pEGYMCR_IncHI2 (GenBank accession number MT499884) using both BlastN and SnapGene 5.2 (Insightful Science, www.snapgene.com) (accessed on 12 January 2021). SnapGene was used for the drawing and annotation of the pEGY49_MCR1.1 plasmid, while PlasmidFinder (https: / / cge.cbs.dtu.dk/ / services/PlasmidFinder/) (accessed on 12 January 2021) was used to detect the plasmid incompatibility (Inc) groups. Insertion sequence (IS) elements of the plasmid were identified using Mobile Genetic Element finder (https: / / cge.cbs.dtu.dk/services/MobileElementFinder/) (accessed on 20 January 2021). The circular image and comparisons between other reported similar plasmids were performed using the BLAST Ring Image Generator (BRIG) tool (http:/ / sourceforge. net/projects/brig) (accessed on 4 February 2021). A comparative analysis of the genetic environment surrounding $\mathrm{mcr}-1.1$ gene with previously reported ones using BlastN was performed and schematic diagrams of the genetic contexts of mcr-1.1 gene were drawn using the SnapGene tool (GSL Biotech LLC, San Diego, CA, USA).

\section{Results and Discussion}

\subsection{Antimicrobial Resistance Profiles and Colistin MIC}

The susceptibility of $67 \mathrm{E}$. coli strains isolated from patients with UTIs to different antibiotics showed that $89.6 \%$ of these isolates possessed an MDR phenotype being resistant to $\geq 3$ groups of antimicrobials. All the isolates were resistant to amoxicillin-clavulanate, third and fourth generation cephalosporins. High resistance rates, exceeding 50\%, were detected for fluoroquinolones, sulfamethoxazole-trimethoprim and gentamicin. While $67.2 \%$ of the isolates were resistant to imipenem, meropenem retained its activity against most of the isolates with a percentage of susceptibility of $86.6 \%$. Meropenem is listed as a precious antibiotic in Egyptian hospital settings and is not widely prescribed in out-patient clinics. The most frequently encountered MDR profile included resistance to amoxicillin-clavulanate, cephalosporins, fluoroquinolones and sulfamethoxazole-trimethoprim (Supplementary Table S1), a profile that correlates with the extensive usage of these antibiotics in Egyptian healthcare establishments as per the Infectious Diseases Society of America (IDSA) guidelines implemented in these facilities. The prevalence of the MDR phenotype among UPEC isolates has been recently reported in different governorates in Egypt. Gawad et al. [2] detected the MDR phenotype among 91\% of UPEC isolates in two governorates in the northeastern part of Egypt. In the capital, Cairo, Abdelkhalik et al. [20] reported an MDR pattern in $80 \%$ of UPEC isolated from women with acute uncomplicated cystitis. In the present study, six isolates (8.9\%) were resistant to colistin. Reports from the Upper Egypt sector revealed a colistin resistance of $32.7 \%, 20.8 \%$ and $23.1 \%$ among UPEC isolates in three different governorates $[11,21]$. After a preliminary screening using disk diffusion method, the MIC of colistin against the 67 tested isolates in the current study was determined by 
broth microdilution, the recommended method by CLSI 2020. The colistin MIC values ranged from 0.125 to $4 \mu \mathrm{g} / \mathrm{mL}$ with $\mathrm{MIC}_{50}$ and $\mathrm{MIC}_{90}$ reaching $2 \mu \mathrm{g} / \mathrm{mL}$ (Figure 1). Using the broth microdilution method, three isolates (4.5\%), EC13049, EC14142 and EC13655, had MIC values $=4 \mu \mathrm{g} / \mathrm{mL}$ and were selected for further tests.

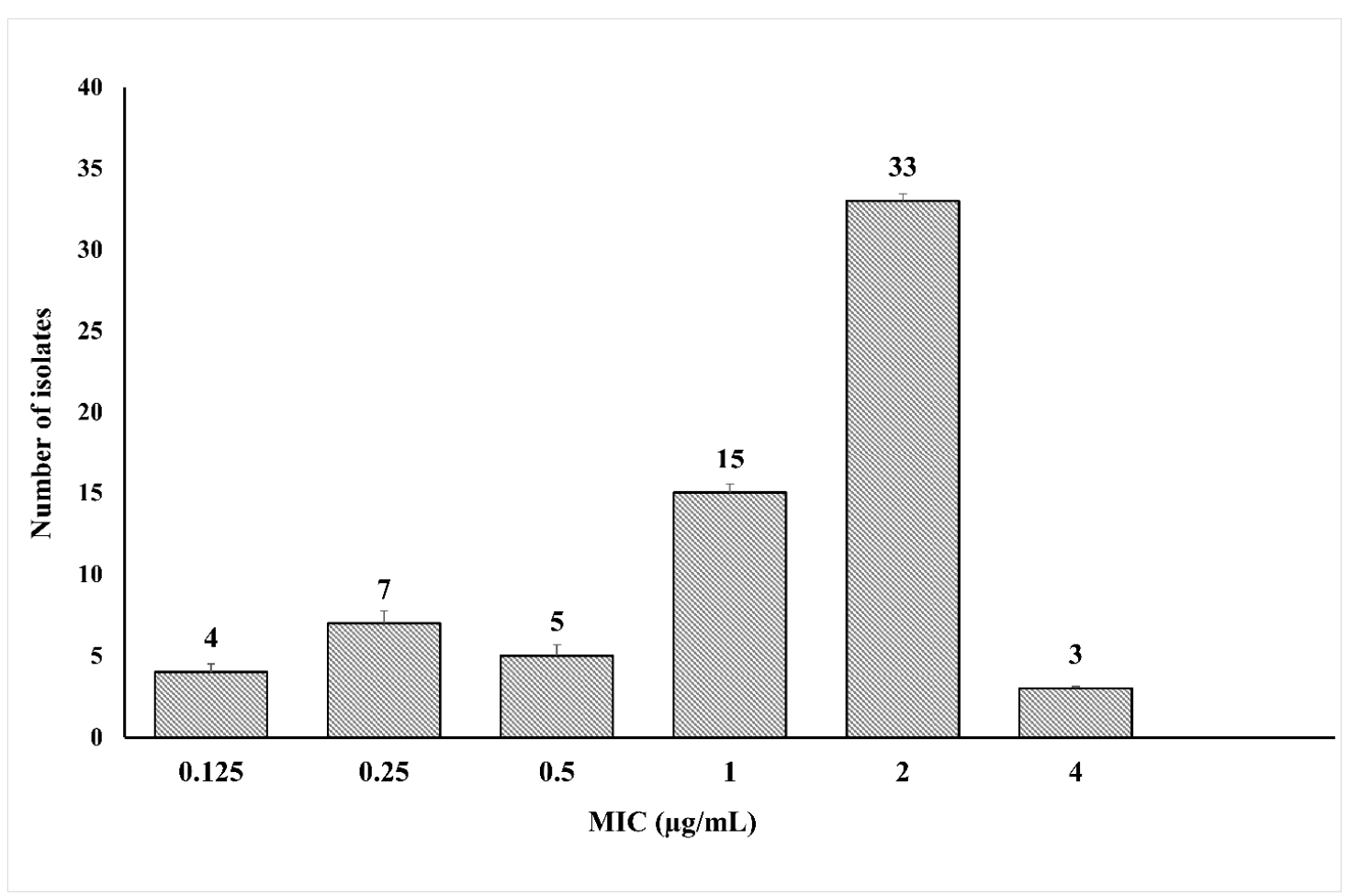

Figure 1. Distribution of colistin MIC values among 67 E. coli isolates from urinary tract infections.

\subsection{Molecular Identification of Resistance Genes}

PCR analysis identified plasmid-mediated colistin resistance associated with the presence of the mcr-1 gene in three MDR isolates, EC13049, EC14142 and EC13655 (Figure 2). None of the isolates harbored the $m c r-2$ gene. Two $m c r-1$-positive clinical E. coli isolates have been identified in Egypt to date; the first one was isolated from a patient with bacteremia while the second was recovered from a cancer patient $[10,11]$. Nevertheless, reports on mcr-1-producing E. coli isolates recovered from livestock or food in Egypt were numerous and included those from a diseased cow [22], healthy broilers [23], cheese [24] and beef sausage [25]. The use of colistin in the Egyptian farming industry as a therapeutic and prophylactic agent is accumulating $m c r-1$ in food-producing animals with a potential risk of its transfer to human food chain [26]. This imposes a challenge in a country struggling with high burden of infectious diseases and low restrictions on antibiotics access as Egypt [22]. The problem of antimicrobial resistance is aggravated when isolates presenting the mcr-1 gene are co-expressing other genes of resistance, such as those encoding extended-spectrum $\beta$-lactamases or carbapenemases [27]. The three $m c r-1$-positive isolates in the present study co-harbored genes conferring resistance to extended-spectrum $\beta$-lactams, bla $a_{S H V}$ and bla Isolate EC13655 possessed the carbapenemase gene $b l a_{N D M}$, whereas no isolates carrying the $q n r B$ gene were detected (Table 2). 


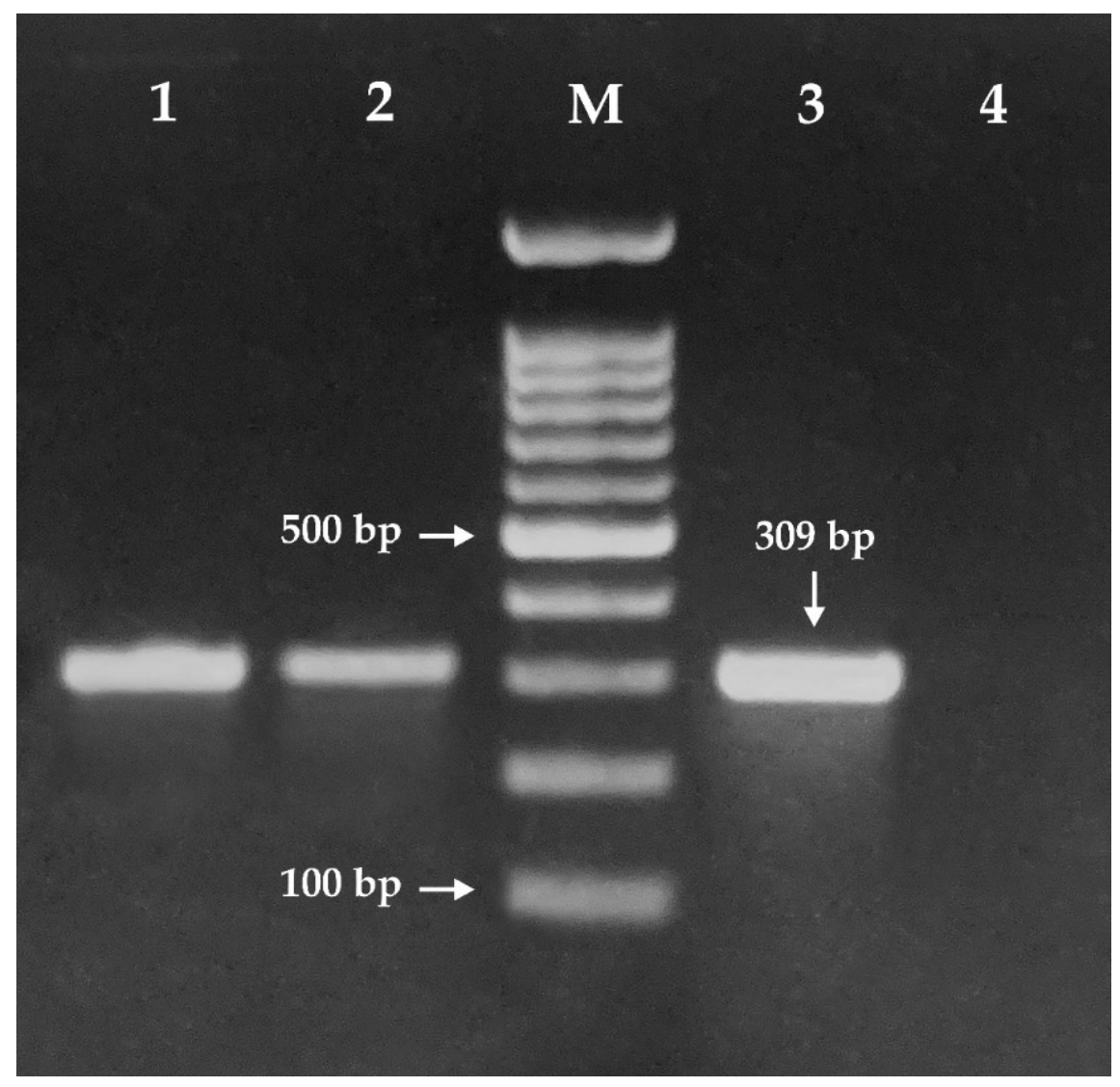

Figure 2. Agarose gel showing PCR amplification of $m c r-1$ gene in three E. coli strains isolated from urinary tract infections. Lane M: DNA molecular weight marker (100 bp ladder). Lanes 1, 2 and 3 show the amplicon (309 bp) of mcr-1 gene corresponding to EC13049, EC14142 and EC13655, respectively. Lane 4 exhibits the negative control (E. coli, ATCC 25922).

Table 2. Antimicrobial resistance profile of colistin-resistant E. coli clinical isolates and their transconjugants.

\begin{tabular}{|c|c|c|c|c|}
\hline E. coli Isolates & $\begin{array}{l}\text { Colistin MIC } \\
\quad(\mu \mathrm{g} / \mathrm{mL})\end{array}$ & $\begin{array}{c}\text { Conjugation } \\
\text { Frequency }^{a} \\
\text { (CFU/Donor Cell) }\end{array}$ & Resistance Genes & Resistance Profile \\
\hline EC13049 & 4 & & $m c r-1, b l a_{S H V}, b l a_{T E M}$ & $\begin{array}{l}\text { AMC, CTX, CAZ, FEP, SXT, } \\
\text { CIP, LEV, CN, DO, CT }\end{array}$ \\
\hline $\begin{array}{c}\text { Transconjugant of } \\
\text { EC13049 }\end{array}$ & 32 & $2.8 \times 10^{-4}$ & $m c r-1, b l a_{S H V}, b l a_{T E M}$ & $\begin{array}{l}\text { AMC, CTX, CAZ, FEP, SXT, } \\
\text { CIP, LEV, CN, DO, CT, RD }\end{array}$ \\
\hline EC14142 & 4 & & $m c r-1, b l a_{S H V}, b l a_{T E M}$ & $\begin{array}{c}\text { AMC, CTX, CAZ, FEP, IPM, } \\
\text { SXT, CIP, LEV, CN, CT }\end{array}$ \\
\hline $\begin{array}{c}\text { Transconjugant of } \\
\text { EC14142 }\end{array}$ & 8 & $5.8 \times 10^{-5}$ & $m c r-1, b l a_{S H V}, b l a_{T E M}$ & $\begin{array}{c}\text { AMC, CTX, CAZ, FEP, IPM, } \\
\text { CIP, LEV, CT, RD }\end{array}$ \\
\hline EC13655 & 4 & & $\begin{array}{c}\text { mcr-1, } \text { bla }_{S H V}, b l a_{T E M} \\
\text { bla }_{N D M}\end{array}$ & $\begin{array}{l}\text { AMC, CTX, CAZ, FEP, IPM, } \\
\text { SXT, CIP, LEV, CN, DO, CT }\end{array}$ \\
\hline $\begin{array}{c}\text { Transconjugant of } \\
\text { EC13655 }\end{array}$ & 16 & $1.7 \times 10^{-7}$ & $\begin{array}{c}m c r-1, \text { bla }_{S H V}, \text { bla }_{T E M}, \\
\text { bla }_{N D M}\end{array}$ & $\begin{array}{l}\text { AMC, CTX, CAZ, FEP, IPM, } \\
\text { SXT, CIP, LEV, CN, CT, RD }\end{array}$ \\
\hline
\end{tabular}

${ }^{a}$ The recipient was rifampicin-resistant $E$. coli $\mathrm{K}-12$ with a colistin MIC of $0.125 \mu \mathrm{g} / \mathrm{mL}$. AMC: amoxicillin-clavulanate; CTX: cefotaxime; CAZ: ceftazidime; FEP: cefepime; IPM: imipenem; SXT: trimethoprim-sulfamethoxazole; CIP: ciprofloxacin; LEV: levofloxacin; CN: gentamicin; DO: doxycycline; CT: colistin, RD: rifampicin. 


\begin{abstract}
3.3. Detection of Amino Acids Alterations in Two-Component Systems; PmrAB and PhoPQ and Their Regulators, MgrB and PmrD

Acquired resistance to colistin is exerted through chromosomal mechanisms modifying the lipid A moiety of Gram-negative bacteria leading to its inactivation and consequent loss of the colistin target. These modifications are associated with mutations inthe twocomponent systems PhoP/PhoQ and PmrA/PmrB [28]. Mutations in $m g r B$ and $p m r D$ genes are also known to play a role in colistin resistance in the Enterobacteriaceae, where the inactivation of $\mathrm{MgrB}$, the negative regulator of the PhoPQ system, leads to overexpression of the $p h o P Q$ operon, whereas the activation of PmrD by PhoP upregulates PmrAB [15]. To address this part, the full nucleotide sequences of $P h o P, P h o Q, p m r A, p m r B, m g r B$ and $p m r D$ from the three colistin-resistant isolates were inspected and compared to those of $E$. coli K-12 MG1655 (Table 3).
\end{abstract}

Table 3. Mutations in chromosomally encoded genes related to colistin resistance in tested E. coli clinical isolates.

\begin{tabular}{|c|c|c|c|c|c|c|}
\hline \multirow{2}{*}{$\begin{array}{l}\text { E. coli } \\
\text { Isolate }\end{array}$} & \multicolumn{6}{|l|}{ Gene/Protein } \\
\hline & pmrA/PmrA & $p m r B / P m r B$ & $p m r D / P m r D$ & PhoP/PhoP & PhoQ/PhoQ & $m g r B / M g r B$ \\
\hline EC13049 & $\mathrm{S} 29 \mathrm{G}^{\mathrm{b}}$ & $\begin{array}{c}\mathrm{H} 2 \mathrm{R}^{\mathrm{b}}, \mathrm{D} 283 \mathrm{G}^{\mathrm{b}} \\
\mathrm{A} 360 \mathrm{~V}^{\mathrm{b}}\end{array}$ & $\begin{array}{l}\text { L19I b, S71C b } \\
\text { V83A }^{b}\end{array}$ & None & None & None \\
\hline EC14142 & $\mathrm{S} 29 \mathrm{G}^{\mathrm{b}}, \mathrm{T} \mathrm{S}^{\mathrm{b}}$ & $\begin{array}{l}\text { H2R }{ }^{b}, \text { E123D }{ }^{a} \\
\text { D283G }\end{array}$ & 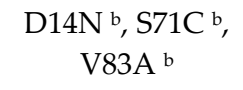 & V108M ${ }^{b}, I_{4} L^{b}$ & $\begin{array}{l}\mathrm{A} 166 \mathrm{~V}^{\mathrm{b}} \\
\mathrm{S} 138 \mathrm{~T}^{\mathrm{b}}\end{array}$ & None \\
\hline EC13655 & S29G b, G144S a & $\mathrm{D} 283 \mathrm{G}^{\mathrm{b}}, \mathrm{Y} 358 \mathrm{~N}^{\mathrm{a}}$ & None & $\mathrm{V}^{108 \mathrm{M}^{\mathrm{b}}}, \mathrm{I} 44 \mathrm{~L}^{\mathrm{b}}$ & $\mathrm{S} 138 \mathrm{~T}^{\mathrm{b}}$ & None \\
\hline
\end{tabular}

a Polymorphism reported to be associated with colistin resistance. ${ }^{b}$ Reported polymorphism but its association with colistin resistance is unknown.

The tested isolates exhibited a wild type $m g r B$. Sequence analysis of the PhoP revealed two missense mutations (I44L and V108M). These variations are frequently reported in $E$. coli as per the available sequences in GenBank, but their impact on colistin resistance is uncomprehended [28]. Amino acid substitutions were found in two sites of PhoQ: A166V and S138T. These two mutations have not been related so far to colistin resistance [28]. The PmrA sequence revealed three amino acid substitutions, among which G144S substitution in isolate EC13655 is reported to contribute to colistin resistance [29]. Six amino acid alterations were detected in PmrB, among which E123D and Y358N mutations, located in the histidine kinase and phosphate-related domain, might affect the phosphate transfer between the two-component systems resulting in colistin-resistant isolates [29]. PmrD, which promotes the connection of PhoPQ and PmrAB two-component systems, displayed mutations at four sites (L19I, S71C, V83A and D14N), but their associations with colistin resistance have not been reported [15]. Although the double mechanism of colistin resistance, the production of $m c r-1$ gene along with amino acid substitution in PmrB (E123D and Y358N) or PmrA (G144S), depicted in this study in isolates EC14142 and EC13655 is considered a rare finding, it has been reported earlier by other investigators [30].

\title{
3.4. Conjugative Transfer of mor-1 Gene
}

Broth mating experiments and subsequent PCR analysis were performed for the mcr-1-positive isolates: EC13049, EC14142 and EC13655. Transconjugants were recovered from the three donors, inferring that $m c r-1$ was positioned on self-transferable conjugative plasmids (Table 2). Conjugation frequencies were in the range of $10^{-4}$ to $10^{-7} \mathrm{CFU}$ per donor cell and the values of colistin MIC for the transconjugants increased from 64- to 256-fold as compared to the recipient. The resistance phenotype and genotype of donors and transconjugants were almost identical except for the failure of transfer of gentamicin and sulfamethoxazole-trimethoprim in EC14142 and doxycycline in isolate EC13655. This suggests that the resistance determinants for these antibiotics might be located on non- 
transferable plasmids. A previous exposure to tetracycline seems to be required to enhance the conjugal transfer of tetracycline resistance determinants as reported earlier [31]. The conjugative transfer of the $m c r-1$ gene and the co-transfer of $b l a_{\mathrm{SHV}}, b l a_{\mathrm{TEM}}$ and $b l a_{\mathrm{NDM}}$ confirmed by PCR illustrate the potential risk of horizontal gene transfer to other Gramnegative bacteria, raising challenges in tailoring an adequate clinical therapy [32].

\subsection{Genome Analysis of UPEC Strain EC13049}

Since the three $m c r-1$-positive E. coli isolates displayed a near-identical antimicrobial resistance phenotype, the EC13049 strain, with the highest conjugation frequency, was chosen for WGS analysis by the Illumina platform. The de novo assembly revealed that the complete genome of E. coli EC13049 comprises a chromosome of 5,553,206bp distributed in 300 contigs with a depth coverage of $198 \times$ and an overall $G+C$ content of $52.4 \%$ and an N50 of 69479 . The assembly statistics generated through WGS are described in Supplementary Table S2. Genotyping of EC13049 indicated that the isolate belongs to the serogroup O23:H4 and to the serotype ST641 according to the MLST (Pasteur) allelic profile, which uses the sequences of the eight house-keeping genes $(\operatorname{din} B, i c d A$, $p a b B, p o l B, p u t P, \operatorname{trp} A, \operatorname{trp} B$, uid $A)$ with assigned allele numbers $(2,237,23,15,10,15$, $10,12)$. ST641, a worldwide clone previously shown to harbor $\mathrm{mcr}$, was reported in different geographical regions including China, Germany, and Columbia [33-35]. Using the sequence data, two virulence determinant-encoding genes, vat and chuA, known to enable an E. coli strain to colonize the urinary bladder producing UTIs, were located on the genome of EC13049, categorizing this isolate as a uropathogenic one [36]. In addition, Clermont phylotyping assigned the isolate to phylogroup D, which is typically affiliated with UTIs [19]. Typing of the fimH (type 1 fimbrial adhesin) gene showed that the isolate carries fimH63 allele, resulting in the clonotype CH45-63. The resistome of the EC13049 displayed an MDR genotype carrying genes responsible for resistance to aminoglycosides

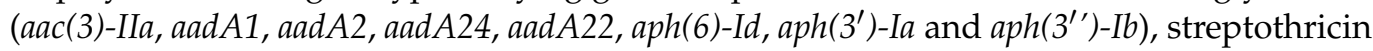
(sat1), amphenicols (floR, catA1, cmlA1), sulphonamides (sul2, sul3), extended-spectrum $\beta$-lactams (bla $\left.a_{\mathrm{SHV}-12}, b l a_{\mathrm{TEM}-1 \mathrm{~B}}\right)$, fluoroquinolones ( $\left.q n r S 1\right)$, colistin $(m c r-1.1)$, tetracycline (tet $A)$, lincosamide $(\operatorname{InuF})$ and trimethoprim (dfrA1). A multidrug efflux pump gene ( $m d f A)$ mediating resistance to aminoglycosides, phenicols, fluoroquinolones, tetracycline, rifamycin and macrolides was detected as well. The expression of MdfA is stated to confer additional resistance to clinically important, chemically unrelated antibiotics even in the absence of other specific genes [30]. Furthermore, a double-serine mutation ( $g y r A$ S83L and parC S80R) and an additional substitution in $g y r A(\mathrm{D} 87 \mathrm{~N})$ associated with resistance to fluoroquinolones were identified by ResFinder. Double-serine substitutions in gyr $A$ and parC have been outlined as a dominant feature of MDR E. coli linked to high resistance levels to fluoroquinolones [37]. PlasmidFinder identified nine plasmid replicon types: $\mathrm{Col}(\mathrm{MG} 828), \mathrm{Col}(\mathrm{pHAD} 28), \mathrm{IncB} / \mathrm{O} / \mathrm{K} / \mathrm{Z}$, IncFIB, IncFIC (FII), IncHI2 (carrying the $\mathrm{mcr}$ 1.1 gene), IncHI2A, IncQ1 and IncX1 (Table 4). 
Table 4. Features, molecular typing, resistance profile and plasmid replicon types carried in UPEC EC13049 isolate from Egypt.

\begin{tabular}{|c|c|c|c|c|c|c|c|}
\hline \multirow[t]{2}{*}{ Serotype $^{a}$} & \multirow[t]{2}{*}{ Phylogroup $^{b}$} & \multirow[t]{2}{*}{ Pathotype $^{c}$} & \multirow{2}{*}{$\begin{array}{c}\text { Sequence } \\
\text { Type } \\
(\mathrm{ST})^{\mathrm{d}}\end{array}$} & \multirow[t]{2}{*}{ Clonotype $^{\mathrm{e}}$} & \multicolumn{2}{|c|}{ Resistance Profile $^{\mathrm{f}}$} & \multirow{2}{*}{$\begin{array}{c}\text { Plasmid } \\
\text { Replicon Type }\end{array}$} \\
\hline & & & & & Antimicrobial Class & $\begin{array}{l}\text { (in black) and Point Mutations } \\
\text { ue) }\end{array}$ & \\
\hline \multirow[t]{13}{*}{$\mathrm{O} 23: \mathrm{H} 4$} & Group D & UPEC & 641 & $\mathrm{CH} 45-63$ & $\beta$-lactams & blasHV-12, bla & Col(MG828) \\
\hline & & & & & Aminoglycosides & $\operatorname{aac}(3)-I I a, a p h\left(3^{\prime}\right)-I a, \operatorname{aad} A 1$, aadA2, & $\mathrm{Col}(\mathrm{pHAD} 28)$ \\
\hline & & & & & & $\begin{array}{l}\text { aadA24, aadA22, aph(6)-Id, } a p h\left(3^{\prime \prime}\right)-I b, \\
m d f A\end{array}$ & IncB/O/K/Z \\
\hline & & & & & Streptothricin & $\begin{array}{l}\text { mafA } \\
\text { sat1 }\end{array}$ & IncFIB \\
\hline & & & & & Phenicol & floR, cat $A 1, \mathrm{cml} A 1, m d f A$ & IncFIC (FII) \\
\hline & & & & & Fluoroquinolones & qnrS1, mdfA, gyrA S83L, gyrA D87N, & IncHI2 \\
\hline & & & & & & parC S80R & IncHI2A \\
\hline & & & & & Colistin & $m c r-1.1$ & IncQ1 \\
\hline & & & & & Folate pathway antagonist & sul2, sul3, dfrA1 & Inc $\times 1$ \\
\hline & & & & & Tetracycline & $\operatorname{tet} A, \operatorname{mdf} A$ & IncX1 \\
\hline & & & & & Lincosamide & $\operatorname{InuF}$ & \\
\hline & & & & & Rifamycin & $m d f A$ & \\
\hline & & & & & Macrolide & $m d f A$ & \\
\hline
\end{tabular}

${ }^{a}$ Data obtained from SerotypeFinder version 2.0. ${ }^{b}$ Based on ClermonTyping method. ${ }^{c}$ Pathotype: as determined by the presence of chuA and vat virulence genes. ${ }^{\mathrm{d}}$ ST: sequence type, data obtained from MLST version 2.0. ${ }^{\mathrm{e}}$ Clonotype: determined according to fumCfimH alleles, data obtained from CHTyper version 1.0. ${ }^{\mathrm{f}}$ ARGs: Antimicrobial resistance genes and point mutations as obtained from ResFinder version 4.0. $\mathrm{g}$ Data represent plasmid incompatibility (Inc) group designations as determined by PlasmidFinder version 2.0. Bold formatting indicates the plasmid carrying the $m c r-1.1$ gene.

\subsection{Genomic Location of mor-1.1 Gene and Similarity to Published Plasmids}

The mor-1.1 gene was located on an IncHI2 plasmid which was 210,842 bp in length and had an average $\mathrm{G}+\mathrm{C}$ content of $45.3 \%$. This is consistent with previous findings indicating that the global spread of $m c r-1$ is mainly generated by three plasmid replicon types: IncI2, IncX4, and IncHI2 [8]. The plasmid designated pEGY49_MCR1.1 (accession number: MW719568) was shown to be a conjugative plasmid transferring the $\mathrm{mcr}-1.1$ gene to the recipient $E$. coli K-12 and was subtyped as pST4. The pEGY49_MCR1.1 plasmid contained 236 CDSs (128 hypothetical proteins) and showed the conserved IncHI2 architecture comprising genes encoding proteins involved in conjugative transfer, replication, plasmid stabilization and maintenance. Furthermore, genes corresponding to Tn3 transposon and to the insertion sequence ISApI1 were located on the plasmid. pEGY49_MCR1.1 carried the tellurium resistance genes (ter $A B C D E$ ) usually associated with IncHI2 plasmids, in addition to terX, ter $Y$ and terW [38]. Transposon Tn21 known to encode genes for heavy-metal resistance, namely mercuric compounds, was found as well on the plasmid [8]. BLASTn analysis of pEGY49_MCR1.1 with the available database showed that this plasmid shared high sequence identity (99.9\% nucleotide identity, 93\% sequence length) with the first reported IncHI2 mcr-1-positive plasmid pHNSHP45-2 (GenBank ID KU341381) identified in China [39]. In comparison with the two IncHI2 sequenced plasmids from Egypt, pEGY49_MCR1.1 showed 100\% nucleotide identity (93\% sequence length) to the IncHI2 pEGY1-MCR-1 (GenBank ID CP023143) plasmid identified in an E. coli strain isolated from a popular Egyptian raw milk cheese, karish cheese [24] and shared high similarity (99.9\% nucleotide identity, $75 \%$ sequence length) to the $m c r-1$-positive IncHI2 plasmid pEGYMCR_IncHI2 (GenBank ID MT499884) identified in E. coli strain recovered from a chicken carcass in the Upper Egypt Sector [8]. The remarkable similarity of the mcr-1carrying plasmid in E. coli identified from human sources in the current study to those recovered from different food origins in Egypt reflects the local epidemic nature of this IncHI2 plasmid that circulates among different sources and facilitates the dissemination of the mcr-1 gene from the food chain to humans. pEGY49_MCR1.1 shared a striking sequence similarity to two IncHI2 mcr-1-bearing plasmids from clinical source identified in the Middle East region, where it was totally identical to pMS8345A (100\% nucleotide identity, $92 \%$ sequence length) recovered from E. coli isolated from a patient in Qatar (GenBank ID CP025402) [40], and was highly similar (99.9\% nucleotide identity, $92 \%$ sequence 
length) to pSA26-MCR1 (GenBank ID KU743384) isolated from E. coli strain from a patient in Saudi Arabia [41]. This similarity points to the possibility of this plasmid being the key vehicle for the spread of the $m c r-1$ gene among E. coli from the Middle East region to North Africa, where Egypt is located. Additional genes were detected alongside mcr-1 and were associated with resistance to aminoglycosides (aadA2), streptothricin (sat1) and amphenicols (cmlA1) (Figure 3A). The co-existence of other antibiotic resistance genes on mcr-1-carrying plasmids presents the hazard of screening colistin-resistant strains under the imposed selective pressure exerted by antibiotics other than colistin [42].

A

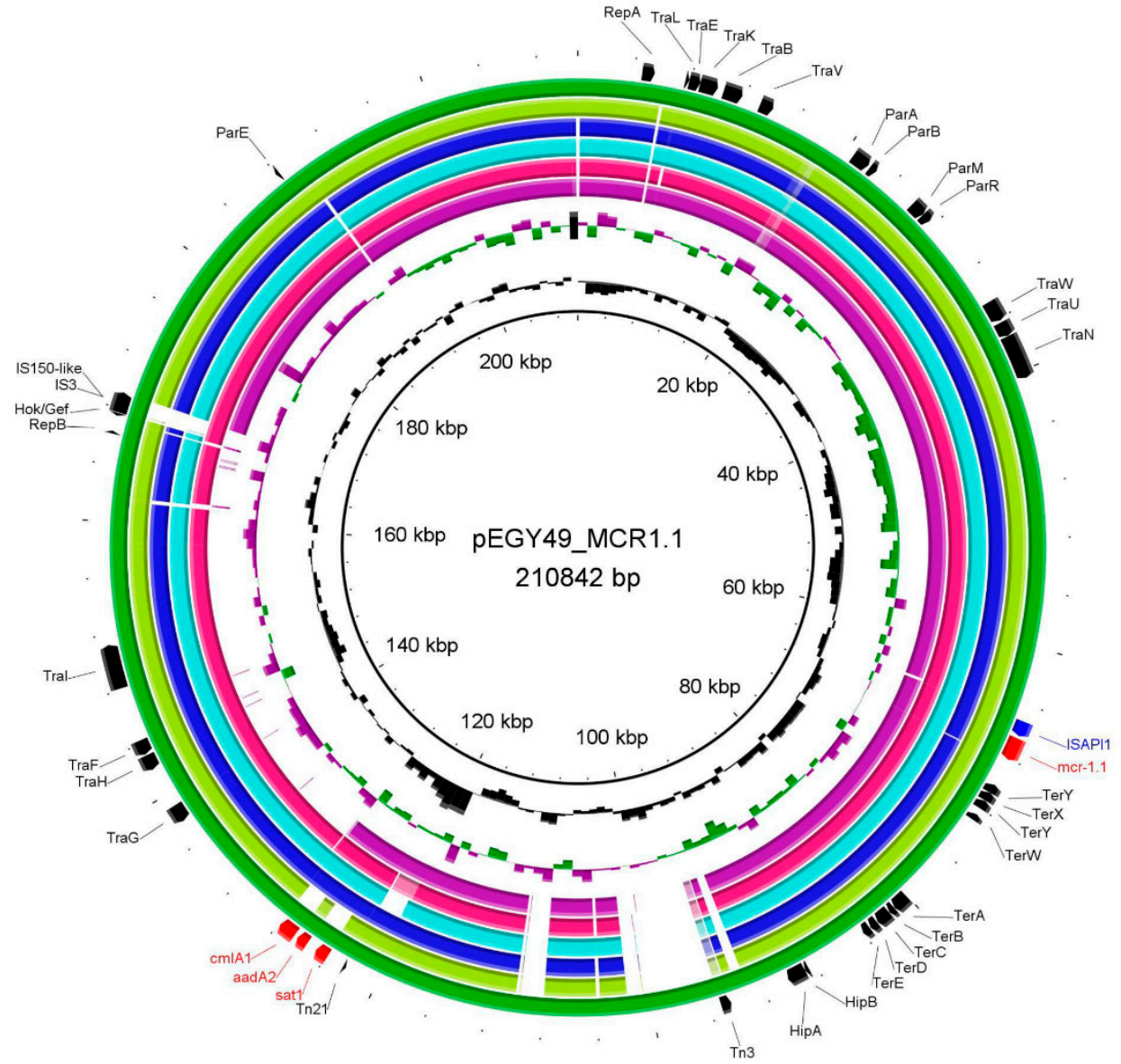

GC Content

GC Skew

GC Skew(-)

GC Skew(+)

pEGYMCR_IncHI2

$100 \%$ identity

$70 \%$ identity

$50 \%$ identity

pHNSHP45-2

$100 \%$ identity

$70 \%$ identity

$50 \%$ identity

pSA26-MCR-1

$100 \%$ identity

$70 \%$ identity

$50 \%$ identity

pEGY1-MCR-1

$100 \%$ identity

$70 \%$ identity

$50 \%$ identity

pMS8345A

$100 \%$ identity

$70 \%$ identity

$50 \%$ identity

pEGY49_MCR1.1

$100 \%$ identity

$70 \%$ identity

$50 \%$ identity

Figure 3. Cont. 


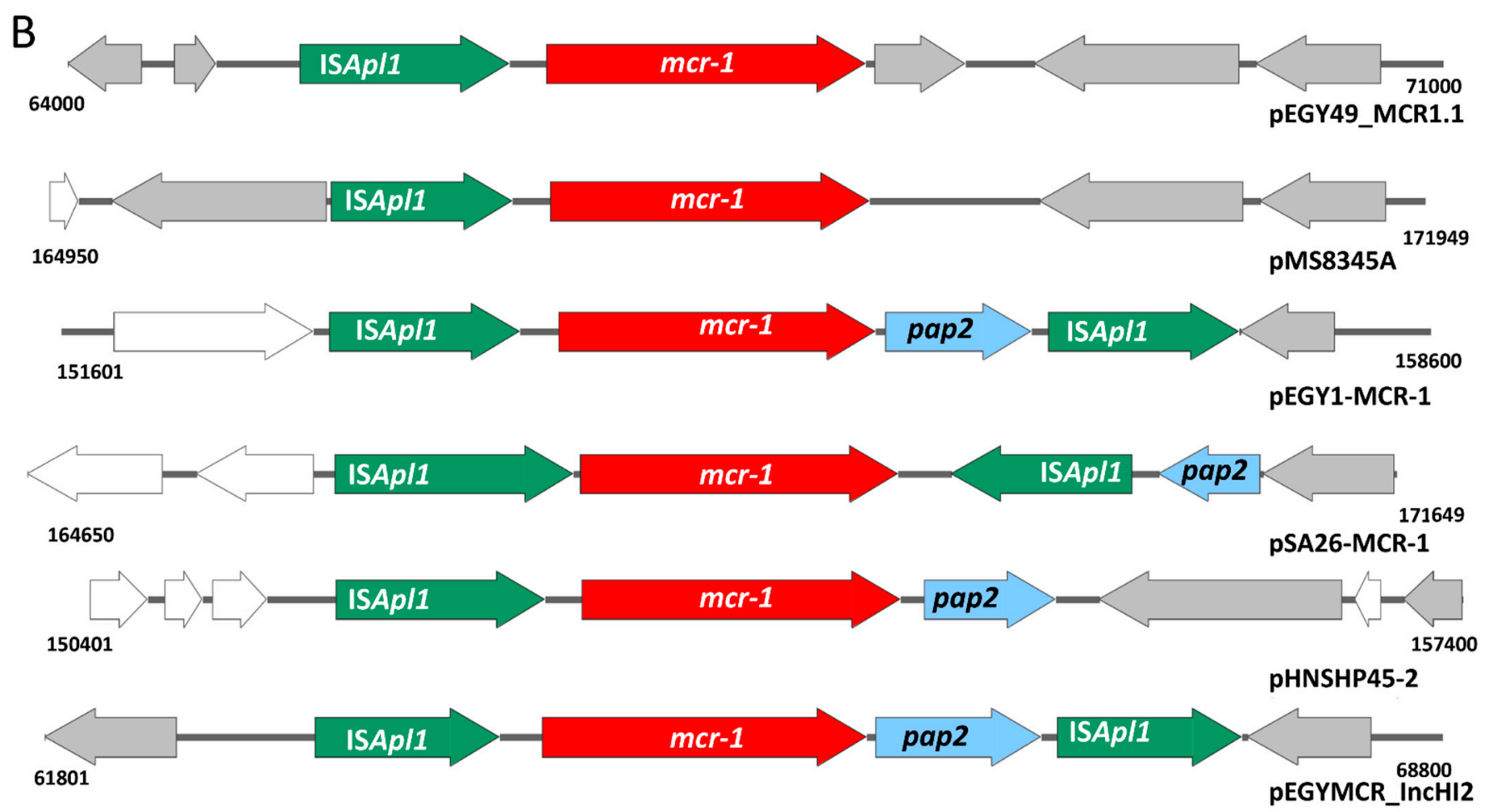

Figure 3. (A) BLAST Ring Image Generator (BRIG) visualization comparing IncHI2-mcr-1-positive E. coli plasmids. The outermost ring (dark green) corresponds to pEGY49_MCR1.1 with its size indicated in the middle of the ring. Next, pMS8345A is shown (light green). The third ring corresponds to pEGY1-MCR-1 (navy blue), then pSA26-MCR1 (aqua) and pHNSHP45-2 (pink). The innermost ring (purple) refers to pEGYMCR_IncHI2. The labels in the outer ring represent the annotation of the genes associated to virulence, antibiotic resistance, stress resistance and replicons. The $m c r-1$ gene and ISApl1 are marked in red and blue, respectively. (B) Genetic environment of $m c r-1$ gene in pEGY49_MCR1.1 compared to those in other IncHI2 plasmids from E. coli. The arrows indicate open reading frames (orf), with red, green, blue, grey and white arrows representing mor-1, ISA 11 element, pap2, ORFs with known function, and ORFs with unknown function, respectively.

\subsection{Genetic Environment of mor-1.1 Gene}

It has been hypothesized that in around 2006, an initial mobilization event of $m c r-1$ occurred via the development of the ISApl1-mor-1-orf-ISApl1 composite transposon [43]. Following this event, the stabilization of $m c r-1$ into a wide range of plasmid types was reached by the loss of flanking ISApl1 elements over time and the subsequent $m c r-1$ spread through plasmid transfer to different bacterial species started. Although the mobilizing capability of the transposon is thought to be suppressed after the loss of both ISApl1 elements, the presence of a single copy is stated to be competent in partial translocation ability, with the upstream copy being more functionally efficient [44]. In silico analysis of the immediate proximity of $m c r-1.1$ in the detected IncHI2 plasmid confirmed that a single upstream copy of the ISApl1 insertion sequence was detected in pEGY49_MCR1.1 (Figure 3B). The absence of the complete composite transposon and the presence of the upstream copy of ISApl1 element suggest that the $m c r-1.1$ gene was transposed into the IncHI2 plasmid not long before isolation [32]. The flanking sequences of mcr-1 in pEGY49_MCR1.1 were highly similar (99.9\% nucleotide identity, $81 \%$ query coverage) to those in plasmid pMS8345A (IncHI2; CP025402), both containing a single upstream copy of ISApl1 element in the direct vicinity of $m c r-1$. The genetic structures surrounding $m c r-1$ gene in pEGY1-MCR-1 (IncHI2; CP023143) and pEGYMCR_IncHI2 (IncHI2; MT499884) showed the presence of ISApl1$m c r-1-o r f-I S A p l 1$ differing from the genetic context of $m c r-1$ gene in the plasmid reported in this study, the latter lacking this composite transposon. In addition to the upstream copy of ISApl1 element in the flanking sequence of mcr-1 in pHNSHP45-2 (IncHI2; KU341381), a pap2-like phosphatase was found directly downstream of mor-1 while pSA26-MCR1 
(IncHI2; KU743384) exhibited a ISApl1-pap2-like phosphatase insertion downstream of mcr1 gene (Figure 3B). Noteworthily, the comparison of the previously mentioned six genetic environments using BLASTn resulted in $99.9 \%$ identity with different query coverage.

\section{Conclusions}

In conclusion, we provide here the first report of the occurrence of an mcr-1-mediated colistin resistance carried on IncHI2 plasmid in an MDR UPEC of O23:H4-ST641 lineage isolated from a patient admitted to a tertiary hospital in Alexandria, Egypt. The notable similarity of this plasmid to the two IncHI2 plasmids from E. coli strains recovered from animal origins described in Egypt so far strongly suggests that this plasmid type is trafficking between food of animal origin and clinical environments disseminating colistin resistance. The unbanned use of colistin as an in-feed antibiotic and growth promoter in Egypt might have created a potential reservoir for the $m c r-1$ gene in food-producing animals that had spread to humans. More proactive regulations to the use of this last-resort drug in the agriculture sector must be implemented to guarantee the success of the treatment and to prevent further dissemination of this resistance.

Supplementary Materials: The following are available online at https://www.mdpi.com/article/ 10.3390/microorganisms9040799/s1, Table S1: Antimicrobial resistance profile for 67 E. coli strains isolated from urinary tract infections where blue boxes represent resistance and white boxes indicate susceptibility to the corresponding antimicrobial disk. Table S2: Assembly statistics generated through WGS of E. coli strain EC 13049.

Author Contributions: Conceptualization, A.S.Z; Data curation, A.S.Z. and N.M.M.; Formal analysis, A.S.Z. and N.M.M.; Funding acquisition, A.S.Z., E.A.E. and N.M.M.; Investigation, A.S.Z. and E.A.E.; Methodology, A.S.Z., E.A.E. and N.M.M.; Writing-original draft, E.A.E. and N.M.M.; Writingreview and editing, A.S.Z., E.A.E. and N.M.M. All authors have read and agreed to the published version of the manuscript.

Funding: This research received no external funding.

Institutional Review Board Statement: Not applicable.

Informed Consent Statement: Not applicable.

Data Availability Statement: The EC13049 whole-genome shotgun sequence was deposited in DDBJ/ENA/GenBank under the BioProject accession number JAENHQ000000000 (https:/ / submit. ncbi.nlm.nih.gov/subs/wgs/JAENHQ000000000 (accessed on 9 April 2021)). The raw sequence data have been submitted to the Sequence Read Archive (SRA)Top of Form (https: / / submit.ncbi. nlm.nih.gov/about/sra/ (accessed on 9 April 2021)) under study accession number PRJNA688626 (https://submit.ncbi.nlm.nih.gov/subs/sra/ PRJNA688626). The plasmid data were deposited in NCBI using Banklt tool under the accession number MW719568.

Acknowledgments: The authors would like to thank Ramy Karam Aziz, Department of Microbiology and Immunology, Center for Microbiome and Genome Research, for providing the E. coli K-12 strain.

Conflicts of Interest: The authors declare no conflict of interest.

\section{References}

1. Terlizzi, M.E.; Gribaudo, G.; Maffei, M.E. UroPathogenic Escherichia coli (UPEC) infections: Virulence factors, bladder responses, antibiotic, and non-antibiotic antimicrobial strategies. Front. Microbiol. 2017, 8, 1566. [CrossRef] [PubMed]

2. Elsayed Gawad, W.; Mohamed Helmy, O.; Mostafa Tawakkol, W.; Mohamed Hashem, A. Antimicrobial resistance, biofilm formation, and phylogenetic grouping of uropathogenic Escherichia coli isolates in Egypt: The role of efflux pump-mediated resistance. Jundishapur J. Microbiol. 2018, 11, e14444. [CrossRef]

3. Kot, B. Antibiotic resistance among uropathogenic Escherichia coli. Polish J. Microbiol. 2019, 68, 403-415. [CrossRef] [PubMed]

4. Schwarz, S.; Johnson, A.P. Transferable resistance to colistin: A new but old threat. J. Antimicrob. Chemother. 2016, 71, 2066-2070. [CrossRef] [PubMed]

5. Emara, M.M.; Abd-Elmonsef, M.M.; Abo Elnasr, L.M.; Elfeky, A.A. Study of $m c r-1$ gene-mediated colistin-resistance in Gramnegative isolates in Egypt. Egypt. J. Med. Microbiol. 2019, 28, 9-16.

6. Zaki, M.E.; ElKheir, N.A.; Mofreh, M. Molecular study of colistin resistant clinical isolates of Enterobacteriaceae species. J. Clin. Mol. Med. 2018, 1, 1-4. [CrossRef] 
7. Liu, Y.Y.; Wang, Y.; Walsh, T.R.; Yi, L.X.; Zhang, R.; Spencer, J.; Doi, Y.; Tian, G.; Dong, B.; Huang, X.; et al. Emergence of plasmid-mediated colistin resistance mechanism MCR-1 in animals and human beings in China: A microbiological and molecular biological study. Lancet Infect. Dis. 2016, 16, 161-168. [CrossRef]

8. Sadek, M.; Ortiz de la Rosa, J.M.; Abdelfattah Maky, M.; Korashe Dandrawy, M.; Nordmann, P.; Poirel, L. Genomic features of MCR-1 and extended-spectrum beta-lactamase-producing Enterobacterales from retail raw chicken in Egypt. Microorganisms 2021, 9, 195. [CrossRef]

9. Yamaguchi, T.; Kawahara, R.; Hamamoto, K.; Hirai, I.; Khong, D.T.; Nguyen, T.N.; Tran, H.T.; Motooka, D.; Nakamura, S.; Yamamoto, Y. High prevalence of colistin-resistant Escherichia coli with chromosomally carried mcr-1 in healthy residents in Vietnam. mSphere 2020, 5, e00117-20. [CrossRef] [PubMed]

10. Elnahriry, S.S.; Khalifa, H.O.; Soliman, A.M.; Ahmed, A.M.; Hussein, A.M.; Shimamoto, T.; Shimamoto, T. Emergence of plasmidmediated colistin resistance gene mcr-1 in a clinical Escherichia coli isolate from Egypt. Antimicrob. Agents Chemother. 2016, 60, 3249-3250. [CrossRef]

11. Mahmoud, A.T.; Salim, M.T.; Ibrahem, R.A.; Gabr, A.; Halby, H.M. Multiple drug resistance patterns in various phylogenetic groups of hospital-acquired uropathogenic E. coli isolated from cancer patients. Antibiotics 2020, 9, 108. [CrossRef]

12. Clinical and Laboratory Standards Institute. Performance Standards for Antimicrobial Susceptibility Testing, 30th ed.; Document M100-S30; CLSI: Wayne, PA, USA, 2020.

13. Magiorakos, A.P.; Srinivasan, A.; Carey, R.T.; Carmeli, Y.; Falagas, M.T.; Giske, C.T.; Harbarth, S.; Hindler, J.T.; Kahlmeter, G.; Olsson-Liljequist, B.; et al. Multidrug-resistant, extensively drug-resistant and pandrug-resistant bacteria: An international expert proposal for interim standard definitions for acquired resistance. Clin. Microbiol. Infect. 2012, 18, 268-281. [CrossRef]

14. Ahmed, Z.S.; Elshafiee, E.A.; Khalefa, H.S.; Kadry, M.; Hamza, D.A. Evidence of colistin resistance genes (mcr-1 and $m c r-2)$ in wild birds and its public health implication in Egypt. Antimicrob. Resist. Infect. Control 2019, 8, 197. [CrossRef]

15. Kim, S.; Woo, J.H.; Kim, N.; Kim, M.H.; Kim, S.Y.; Son, J.H.; Moon, D.C.; Lim, S.K.; Shin, M.; Lee, J.C. Characterization of chromosome-mediated colistin resistance in Escherichia coli isolates from livestock in Korea. Infect. Drug Resist. 2019, 12, 3291-3299. [CrossRef]

16. Ibrahim, N.H.; Alqahtani, F.Y.; Aleanizy, F.S.; Alfaraj, R.; Mahmoud, A.Z.; Zakaria, A.S. Frequency of plasmid-mediated quinolone resistance determinants Qnr and QepA among clinical isolates of Escherichia coli and Klebsiella pneumoniae producing extended-spectrum $\beta$-lactamases from Saudi Arabia intensive care units. Int. J. Microbiol. Res. 2017, 9, $924-929$.

17. Hatrongjit, R.; Kerdsin, A.; Akeda, Y.; Hamada, S. Detection of plasmid-mediated colistin-resistant and carbapenem-resistant genes by multiplex PCR. MethodsX 2018, 5, 532-536. [CrossRef] [PubMed]

18. Franco, A.; Leekitcharoenphon, P.; Feltrin, F.; Alba, P.; Cordaro, G.; Iurescia, M.; Tolli, R.; D'Incau, M.; Staffolani, M.; Di Giannatale, E.; et al. Emergence of a clonal lineage of multidrug-resistant ESBL-producing Salmonella infantis transmitted from broilers and broiler meat to humans in Italy between 2011 and 2014. PLoS ONE 2015, 10, e0144802. [CrossRef]

19. Clermont, O.; Bonacorsi, S.; Bingen, E. Rapid and simple determination of the Escherichia coli phylogenetic group. Appl. Environ. Microbiol. 2000, 66, 4555-4558. [CrossRef]

20. Abdelkhalik, A.M.; Agha, M.M.; Zaki, A.M.; Tahoun, A.T. Clinical and lab-assessed antibiotic resistance pattern of uropathogens among women with acute uncomplicated cystitis. Egypt. J. Hosp. Med. 2018, 73, 7860-7868. [CrossRef]

21. El-Mokhtar, M.A.; Mandour, S.A.; Shahat, A.A. Colistin resistance among multidrug-resistant E. coli isolated from Upper Egypt. Egypt. J. Med. Microbiol. 2019, 28, 11-17.

22. Khalifa, H.O.; Ahmed, A.M.; Oreiby, A.F.; Eid, A.M.; Shimamoto, T.; Shimamoto, T. Characterisation of the plasmid-mediated colistin resistance gene $m c r-1$ in Escherichia coli isolated from animals in Egypt. Int. J. Antimicrob. Agents 2016, 47, 413-414. [CrossRef]

23. Moawad, A.A.; Hotzel, H.; Neubauer, H.; Ehricht, R.; Monecke, S.; Tomaso, H.; Hafez, H.M.; Roesler, U.; El-Adawy, H. Antimicrobial resistance in Enterobacteriaceae from healthy broilers in Egypt: Emergence of colistin-resistant and extendedspectrum $\beta$-lactamase-producing Escherichia coli. Gut Pathog. 2018, 10, 39. [CrossRef]

24. Hammad, A.M.; Hoffmann, M.; Gonzalez-Escalona, N.; Abbas, N.H.; Yao, K.; Koenig, S.; Allué-Guardia, A.; Eppinger, M. Genomic features of colistin resistant Escherichia coli ST69 strain harboring mcr-1 on IncHI2 plasmid from raw milk cheese in Egypt. Infect. Genet. Evol. 2019, 73, 126-131. [CrossRef]

25. Sadek, M.; Poirel, L.; Nordmann, P.; Nariya, H.; Shimamoto, T.; Shimamoto, T. Draft genome sequence of an $m c r-1 /$ Incl2-carrying multidrug-resistant Escherichia coli B1:ST101 isolated from meat and meat products in Egypt. J. Glob. Antimicrob. Resist. 2020, 20, 41-42. [CrossRef] [PubMed]

26. Lima Barbieri, N.; Nielsen, D.W.; Wannemuehler, Y.; Cavender, T.; Hussein, A.; Yan, S.G.; Nolan, L.K.; Logue, C.M. mcr-1 identified in Avian Pathogenic Escherichia coli (APEC). PLoS ONE 2017, 12, e0172997. [CrossRef] [PubMed]

27. Dandachi, I.; Chaddad, A.; Hanna, J.; Matta, J.; Daoud, Z. Understanding the epidemiology of multi-drug resistant Gram-negative bacilli in the Middle East using a one health approach. Front. Microbiol. 2019, 10, 1941. [CrossRef] [PubMed]

28. Delannoy, S.; Le Devendec, L.; Jouy, E.; Fach, P.; Drider, D.; Kempf, I. Characterization of colistin-resistant Escherichia coli isolated from diseased pigs in France. Front. Microbiol. 2017, 8, 2278. [CrossRef] [PubMed] 
29. Sato, T.; Shiraishi, T.; Hiyama, Y.; Honda, H.; Shinagawa, M.; Usui, M.; Kuronuma, K.; Masumori, N.; Takahashi, S.; Tamura, Y.; et al. Contribution of novel amino acid alterations in PmrA or PmrB to colistin resistance in $m c r$-negative Escherichia coli clinical isolates, including major multidrug-resistant lineages O25b:H4-ST131-H30Rx and non-x. Antimicrob. Agents Chemother. 2018, 62, e00864-18. [CrossRef] [PubMed]

30. García-Meniño, I.; Díaz-Jiménez, D.; García, V.; de Toro, M.; Flament-Simon, S.C.; Blanco, J.; Mora, A. Genomic characterization of prevalent mcr-1, mcr-4, and mcr-5 Escherichia coli within swine enteric colibacillosis in Spain. Front. Microbiol. 2019, 10, 2469. [CrossRef]

31. Johnson, T.J.; Singer, R.S.; Isaacson, R.E.; Danzeisen, J.L.; Lang, K.; Kobluk, K.; Rivet, B.; Borewicz, K.; Frye, J.G.; Englen, M.; et al. In Vivo transmission of an IncA/C plasmid in Escherichia coli depends on tetracycline concentration, and acquisition of the plasmid results in a variable cost of fitness. Appl. Environ. Microbiol. 2015, 81, 3561-3570. [CrossRef]

32. Wang, R.; van Dorp, L.; Shaw, L.P.; Bradley, P.; Wang, Q.; Wang, X.; Jin, L.; Zhang, Q.; Liu, Y.; Rieux, A.; et al. The global distribution and spread of the mobilized colistin resistance gene mcr-1. Nat. Commun. 2018, 9, 1179. [CrossRef]

33. Liu, X.; Li, R.; Zheng, Z.; Chen, K.; Xie, M.; Chan, E.W.C.; Geng, S.; Chen, S. Molecular characterization of Escherichia coli isolates carrying $m c r-1$, fos $A 3$, and extended-spectrum-beta-lactamase genes from food samples in China. Antimicrob. Agents Chemother. 2017, 61, e00064-17. [CrossRef]

34. Pulss, S.; Semmler, T.; Prenger-Berninghoff, E.; Bauerfeind, R.; Ewers, C. First report of an Escherichia coli strain from swine carrying an OXA-181 carbapenemase and the colistin resistance determinant MCR-1. Int. J. Antimicrob. Agents 2017, 50, 232-236. [CrossRef]

35. Wise, M.G.; Estabrook, M.A.; Sahm, D.F.; Stone, G.G.; Kazmierczak, K.M. Prevalence of mcr-type genes among colistin-resistant Enterobacteriaceae collected in 2014-2016 as part of the INFORM global surveillance program. PLoS ONE 2018, 13, e0195281. [CrossRef]

36. Kubelová, M.; Koláčková, I.; Gelbíčová, T.; Florianová, M.; Kalová, A.; Karpíšková, R. Virulence properties of mcr-1-positive Escherichia coli isolated from retail poultry meat. Microorganisms 2021, 9, 308. [CrossRef] [PubMed]

37. Fuzi, M.; Szabo, D.; Csercsik, R. Double-serine fluoroquinolone resistance mutations advance major international clones and lineages of various multi-drug resistant bacteria. Front. Microbiol. 2017, 8, 2261. [CrossRef]

38. Whelan, K.F.; Sherburne, R.K.; Taylor, D.E. Characterization of a region of the IncHI2 plasmid R478 which protects Escherichia coli from toxic effects specified by components of the tellurite, phage, and colicin resistance cluster. J. Bacteriol. 1997, $179,63-71$. [CrossRef]

39. Zhi, C.; Lv, L.; Yu, L.F.; Doi, Y.; Liu, J.H. Dissemination of the mcr-1 colistin resistance gene. Lancet Infect. Dis. 2016, 16, 292-293. [CrossRef]

40. Forde, B.M.; Zowawi, H.M.; Harris, P.N.; Roberts, L.; Ibrahim, E.; Shaikh, N.; Deshmukh, A.; Ahmed, M.A.S.; Al Maslamani, M.; Cottrell, K.; et al. Discovery of mcr-1-mediated colistin resistance in a highly virulent Escherichia coli lineage. $m$ Sphere 2018, 3, e00486-18. [CrossRef] [PubMed]

41. Sonnevend, Á.; Ghazawi, A.; Alqahtani, M.; Shibl, A.; Jamal, W.; Hashmey, R.; Pal, T. Plasmid-mediated colistin resistance in Escherichia coli from the Arabian Peninsula. Int. J. Infect. Dis. 2016, 50, 85-90. [CrossRef]

42. Anyanwu, M.U.; Okpala, C.O.R.; Chah, K.F.; Shoyinka, V.S. Prevalence and traits of mobile colistin resistance gene harbouring isolates from different ecosystems in Africa. Biomed. Res. Int. 2021, 2021, 6630379. [CrossRef] [PubMed]

43. Poirel, L.; Kieffer, N.; Brink, A.; Coetze, J.; Jayol, A.; Nordmann, P. Genetic features of MCR-1-producing colistin-resistant Escherichia coli isolates in South Africa. Antimicrob. Agents Chemother. 2016, 60, 4394-4397. [CrossRef] [PubMed]

44. Snesrud, E.; He, S.; Chandler, M.; Dekker, J.P.; Hickman, A.B.; McGann, P.; Dyda, F. A Model for transposition of the colistin resistance gene mcr-1 by ISApl1. Antimicrob. Agents Chemother. 2016, 60, 6973-6976. [CrossRef] [PubMed] 